\title{
LEFT LOOPS WHICH SATISFY THE LEFT BOL IDENTITY
}

\author{
B. L. SHARMA
}

ABSTRACT. It is our purpose in this paper to initiate a study of the algebraic properties of a left loop $Q(\cdot)$ satisfying the identical relation

$$
y(z \cdot y x)=(y \cdot z y) x
$$

for all $x, y, z \in Q$. It is shown that (1) implies right division in $Q(\cdot)$. By introducing a new operation ' $\circ$ ' in $Q$, the connection between the left loop $Q(\cdot)$ and Bol loop $Q(\circ)$ is established. Further we show that the role of nuclei in the left loop theory is not the same as that in the loop theory. We conclude the paper by describing situations in which the left loop $Q(\cdot)$ is Moufang.

1. Introduction. A loop $Q(\cdot)$ is a Bol loop if and only if

$$
(x y \cdot z) y=x(y z \cdot y)
$$

for all $x, y, z \in Q$. Bol loops had their origin in the work of Bol [1] and their algebraic properties have been discussed by Robinson [2]. There exists a duality between those loops satisfying (1) and those loops for which

$$
y(z \cdot y x)=(y \cdot z y) x
$$

for all $x, y, z \in Q$. (1) and (2) are called right Bol identity and left Bol identity respectively. A loop for which (1) ((2)) holds is called a loop with R.B.P. (L.B.P.).

In this paper we discuss the algebraic properties of a left loop $Q(\cdot)$ with L.B.P. We show that (2) implies right division in $Q(\cdot)$. Secondly we introduce a new operation '。' in $Q$ and prove that $Q(\circ)$ is a loop with L.B.P. Robinson [2] has proved results for the loops with R.B.P., so similar theorems hold for the loop $Q(\circ)$ and this fact helps us in discussing results for $Q(\cdot)$. Further we emphasize some principal distinction between the left loop theory and the loop theory. Namely, such notions as nuclei are not valid in the left loop theory. Instead, some new notions introduced are more effective in the case of a left loop with L.B.P. We conclude the paper by describing situations in which a

Received by the editors December 11, 1973 and, in revised form, December 23, 1975.

AMS (MOS) subject classifications (1970). Primary 20 N05.

Key words and phrases. Left loop, Bol loop, Moufang loop, left (right) Bol property, nucleus, automorphism, left inverse property. 
left loop with L.B.P. is Moufang. We recall that a left quasi-group is a magma (groupoid) $Q(\cdot)$ in which the equation $a \cdot x=b$ is uniquely soluble: $x=a \backslash b$. A left quasi-group $Q(\cdot)$ with right identity $\varepsilon(a \cdot \varepsilon=a, \forall a \in Q)$ is called a left loop.

In this paper we denote the right inverse of each $x \in Q$ by $x^{\rho}$, i.e., $x \cdot x^{\rho}=\varepsilon$.

2. TheOREM 1. If $Q(\cdot)$ is a left loop with L.B.P., then

(i) $Q(\cdot)$ satisfies the left inverse property,

(ii) $(x \cdot y x)^{\rho}=x^{\rho} \cdot y^{\rho} x^{\rho}$ for all $x, y \in Q$.

Proof. (i) Let $y=z^{\rho}$ in (2); then $z^{\rho}\left(z \cdot z^{\rho} x\right)=\left(z^{\rho} \cdot z z^{\rho}\right) x=z^{\rho} x$ for all $x, z \in Q$. Thus, by the left cancellative law, we have

$$
z \cdot z^{\rho} x=x \text { for all } x, z \in Q
$$

Let $z=y^{\rho}$ in (2); then $y\left(y^{\rho} \cdot y x\right)=\left(y \cdot y^{\rho} y\right) x=y x$ by (3), for all $x, y \in Q$. Thus, by the left cancellative law, we have

$$
z^{\rho} \cdot z x=x \text { for all } x, z \in Q .
$$

This completes the proof of (i). Let $x=\varepsilon$ in (4); then $z^{\rho} \cdot z=\varepsilon$ or

$$
\left(z^{\rho}\right)^{\rho}=z \quad \text { for all } z \in Q
$$

Further, $\varepsilon \cdot \varepsilon=\varepsilon \Rightarrow \varepsilon^{\rho}=\varepsilon$. Let $z=\varepsilon$ in (4); then

$$
\varepsilon \cdot \varepsilon x=x \text { for all } x \in Q .
$$

(ii) For all $x, y \in Q$,

$$
\begin{aligned}
\left(x^{\rho} \cdot y^{\rho} x^{\rho}\right)(x \cdot y x) & =x^{\rho}\left[y^{\rho}\left(x^{\rho}(x \cdot y x)\right)\right] \text { by }(2) \\
& =x^{\rho}\left(y^{\rho} \cdot y x\right) \text { by }(4) \\
& =x^{\rho} x \quad \text { by }(4) \\
& =\varepsilon .
\end{aligned}
$$

So, $(x \cdot y x)^{\rho}=x^{\rho} \cdot y^{\rho} x^{\rho}$ for all $x, y \in Q$. This completes the proof of (ii).

Theorem 2. If $Q(\cdot)$ is a left loop with L.B.P., then for given $a, b \in Q$, the equation $x \cdot a=b$ has a unique solution for $x$ in $Q(\cdot)$.

Proof. Let $x=y^{\rho}$ in (2); then

$$
y \cdot z=(y \cdot z y) y^{\rho} \text { for all } y, z \in Q .
$$

Let us consider the equation

$$
x \cdot a=b \text { for given } a, b \in Q .
$$

If a solution for $x$ exists, then 


$$
\begin{aligned}
a \cdot x & =(a \cdot x a) a^{\rho} \quad \text { by }(7) \\
& =a b \cdot a^{\rho} \quad \text { by }(8)
\end{aligned}
$$

Thus $x=a \backslash a b \cdot a^{\rho}=a^{\rho}\left(a b \cdot a^{\rho}\right)$. Substituting the value of $x$ in (8), we have

$$
\begin{aligned}
{\left[a^{\rho}\left(a b \cdot a^{\rho}\right)\right] a } & =a^{\rho} \cdot a b \quad \text { by }(7) \\
& =b \quad \text { by }(4) .
\end{aligned}
$$

Thus $x \cdot a=b$ has a unique solution for $x$ in $Q(\cdot)$.

We define a new operation ' $\circ$ ' in the left loop $Q(\cdot)$ as follows.

$$
x \circ y={ }_{\operatorname{def}} x \cdot L_{\varepsilon}^{-1} y
$$

where $L_{x} y:=x y$.

LEMMA 1. The operator $L_{\varepsilon}$ is an involutive automorphism in the left loop $Q(\cdot)$ with L.B.P.

Proof. By equation $(6), \varepsilon \cdot \varepsilon x=x$ for all $x \in Q$. Therefore, $L_{\varepsilon}\left(L_{\varepsilon} x\right)=x$, $\left(L_{\varepsilon} L_{\varepsilon}\right)(x)=x$ implies

$$
L_{\varepsilon}=L_{\varepsilon}^{-1}
$$

Let $y=\varepsilon$ in (2); then $\varepsilon(z \cdot \varepsilon x)=\varepsilon z \cdot x$ for all $x, z \in Q$. Thus

$$
L_{\varepsilon}\left(z \cdot L_{\varepsilon} x\right)=L_{\varepsilon} z \cdot x
$$

Putting $w=L_{\varepsilon} x \Rightarrow x=L_{\varepsilon}^{-1} w=L_{\varepsilon} w$ in (11), we have

$$
L_{\varepsilon}(z \cdot w)=\left(L_{\varepsilon} z\right) \cdot\left(L_{\varepsilon} w\right) \text { for all } z, w \in Q .
$$

This completes the proof of Lemma 1.

COROLlARY 1. The operator $L_{\varepsilon}$ is an involutive automorphism of $Q(\circ)$.

Proof. We consider

$$
\begin{aligned}
L_{\varepsilon}(x \circ y) & =L_{\varepsilon}(x \cdot \varepsilon y) \quad \text { by }(9) \text { and }(10) \\
& =\left(L_{\varepsilon} x\right) \cdot y \quad \text { by }(12) \text { and }(6) \\
& =\left(L_{\varepsilon} x\right) \circ(\varepsilon y) \\
& =\left(L_{\varepsilon} x\right) \circ\left(L_{\varepsilon} y\right) \text { for all } x, y \in Q .
\end{aligned}
$$

This completes the proof of Corollary 1 .

COROLlARY 2. The operator $L_{\varepsilon}$ satisfies the property

$$
L_{\varepsilon}\left(x^{\rho}\right)=\left(L_{\varepsilon} x\right)^{\rho} \quad \text { for all } x \in Q .
$$


Proof. For all $x \in Q, x \cdot x^{\rho}=\varepsilon$; thus $L_{\varepsilon}\left(x \cdot x^{\rho}\right)=L_{\varepsilon}(\varepsilon)$. By using (9) and (12), we have

$$
\left(L_{\varepsilon} x\right) \cdot\left(L_{\varepsilon} x^{\rho}\right)=\varepsilon \Rightarrow L_{\varepsilon}\left(x^{\rho}\right)=\left(L_{\varepsilon} x\right)^{\rho} .
$$

This completes the proof of Corollary 2.

THEOREM 3. $Q(\circ)$ is a loop with L.B.P. and having $\varepsilon$ as two-sided identity.

Proof. By definition (9), for all $x, y \in Q, x \circ y=x \cdot \varepsilon y=z \in Q(\circ)$. Consider the equation $a \circ x=b$ for all $a, b \in Q, a \circ x=a \cdot \varepsilon x=b \Rightarrow \varepsilon x$ $=a \backslash b$ or $x=\varepsilon \cdot a^{\rho} b \Rightarrow Q(\circ)$ has a unique left division. Now we consider the equation $x \circ a=b$ for all $a, b \in Q, x \circ a=x \cdot \varepsilon a=b \Rightarrow x=b \cdot(\varepsilon a)^{\rho}$ by Theorem $2=b \cdot \varepsilon a^{\rho}$ by $(13) \Rightarrow Q(\circ)$ has a unique right division. Next we consider, for all $x \in Q, \varepsilon \circ x=\varepsilon \cdot \varepsilon x=x$ by (6), also $x \circ \varepsilon=x \cdot \varepsilon \varepsilon=x$. Thus $\varepsilon$ acts as two-sided identity. Thus we have proved that $Q(\circ)$ is a loop. Next we prove that $Q(\circ)$ has L.B.P.

For all $x, y, z \in Q$,

$$
\begin{aligned}
y \circ(z \circ(y \circ x)) & =y(\varepsilon(z(\varepsilon(y \cdot \varepsilon x))))=y(\varepsilon(z(y \cdot \varepsilon x))) \\
& =(y(\varepsilon z \cdot y)) \cdot(\varepsilon x)=(y(\varepsilon(z \cdot \varepsilon y))) \cdot(\varepsilon x) \\
& =(y \circ(z \circ y)) \circ x .
\end{aligned}
$$

Thus,

$$
y \circ(z \circ(y \circ x))=(y \circ(z \circ y)) \circ x \text { for all } x, y, z \in Q .
$$

Hence the loop $Q(\circ)$ has L.B.P.

THEOREM 4. If $Q(\circ)$ is a loop with L.B.P. and $L$ is an arbitrary involutive automorphism of $Q(\circ)$, then $Q(\cdot)$ equipped with the multiplication $x \cdot y={ }_{\operatorname{def}} x$ $\circ$ Ly is a left loop with L.B.P. and $L$ is an involutive automorphism of $Q(\cdot)$.

Proof. Since $L$ is an involutive automorphism of $Q(\circ)$ and

$$
x \cdot y=_{\mathrm{def}} x \circ L y \text { for all } x, y \in Q,
$$

there exists left division and right division in $Q(\cdot)$. Let $x=e($ identity of $Q(\circ))$ in (14); then

$$
L y=e \cdot y \text { for all } y \in Q .
$$

If we put $y=e$ in (15), we have

$$
L e=e \text {. }
$$

Since $L$ is an involutive automorphism of $Q(\circ)$, we have $L=L^{-1}$. Secondly,

$$
L(x \cdot y)=L(x \circ L y)=(L x) \circ L(L y)=(L x) \circ y=(L x) \cdot(L y) .
$$


Thus,

$$
L(x \cdot y)=L x \cdot L y \quad \text { for all } x, y \in Q .
$$

We have shown that $L$ is an involutive automorphism of $Q(\cdot)$. Now we consider

$$
\begin{aligned}
y(z \cdot y x) & =y \circ L(z \circ L(y \circ L x))=y \circ(L z \circ(y \circ L x)) \\
& =(y \circ(L z \circ y)) \circ L x \quad \text { by }(2) \\
& =(y \circ L(z \circ L y)) \circ L x=(y \cdot z y) x .
\end{aligned}
$$

Thus $Q(\cdot)$ satisfies the L.B.P. This completes the proof of the theorem.

THEOREM 5. If $Q(\circ)$ is a loop with identity $e$ and L.B.P., and $Q(\cdot)$ is a left loop with right identity $\varepsilon$ and L.B.P., such that $x \circ L y=x \cdot y$ and $e=\varepsilon$, then $L$ is an involutive automorphism of $Q(\circ)$ and $Q(\cdot)$.

Proof. For all $x, y \in Q$,

$$
x \circ L y=x \cdot y .
$$

Let $x=e=\varepsilon$ in (17); then

$$
L y=\varepsilon \cdot y \quad \text { for all } y \in Q .
$$

Since $Q(\cdot)$ is a left loop with L.B.P. and by Lemma 1 , it follows that $L$ is an involutive automorphism of $Q(\cdot)$. Now (17) can be written as follows:

$$
x \circ y=x \cdot L y \text { for all } x, y \in Q .
$$

By Corollary 1 of Lemma 1 it follows that $L$ is an involutive automorphism of $Q(\circ)$. This completes the proof of the theorem.

Following Bruck [3, p. 250] the left nucleus $N_{\lambda}$, the middle nucleus $N_{\mu}$ and the right nucleus $N_{\rho}$ of a loop $G(\cdot)$ are defined by:

$$
\begin{aligned}
& N_{\lambda}(\cdot)=\{\text { all } x \in G \mid x \cdot y z=x y \cdot z, \text { all } y, z \in G\}, \\
& N_{\mu}(\cdot)=\{\text { all } y \in G \mid x \cdot y z=x y \cdot z, \text { all } x, z \in G\}, \\
& N_{\rho}(\cdot)=\{\text { all } z \in G \mid x \cdot y z=x y \cdot z, \text { all } x, y \in G\} .
\end{aligned}
$$

Further, Bruck [3, Theorem 2A, p. 255] has proved that each subset $N_{\lambda}(\cdot)$, $N_{\mu}(\cdot), N_{\rho}(\cdot)$ is an associative subloop (group) of $G(\cdot)$. Thus each subset $N_{\lambda}(\circ), N_{\mu}(\circ)$ and $N_{\rho}(\circ)$ of $Q(\circ)$ is an associative subloop of $Q(\circ)$. But we cannot assert for example, that $N_{\lambda}(\cdot)$ is an associative subloop for any left loop $Q(\cdot)$. This fact suggests the following theorem for the left loop $Q(\cdot)$. 
THEOREM 6. If $Q(\cdot)$ is a left loop with L.B.P., then there exist sub-left loops

(a) $Q_{\lambda}(\cdot)=\{$ all $a \in Q \mid a x \cdot \varepsilon y=a \cdot x y$ for all $x, y \in Q\}$,

(b) $Q_{\mu}(\cdot)=\{$ all $a \in Q \mid x a \cdot \varepsilon y=x \cdot$ ay for all $x, y \in Q\}$,

(c) $Q_{\rho}(\cdot)=\{$ all $a \in Q \mid x y \cdot \varepsilon a=x \cdot y a$ for all $x, y \in Q\}$,

of $Q(\cdot)$ such that $N_{\lambda}(\cdot) \cap Q_{\lambda}(\cdot)=\varnothing, N_{\mu}(\cdot) \cap Q_{\mu}(\cdot)=\varnothing$ and $N_{\rho}(\cdot) \subset Q_{\rho}(\cdot)$, where $\varnothing$ denotes the empty set.

Proof. If $a, b$ are two elements of $Q_{\lambda}(\cdot)$ and $x, y$ any two elements of $Q(\cdot)$, then

$$
\begin{aligned}
a b \cdot x y & =a b \cdot \varepsilon(\varepsilon x \cdot \varepsilon y)=a(b(\varepsilon \cdot x y)) \quad \text { by }(\mathrm{a}) \\
& =a(b(\varepsilon x \cdot \varepsilon y))=a((b \cdot \varepsilon x) y) \\
& =(a(b \cdot \varepsilon x)) \cdot(\varepsilon y)=(a b \cdot x) \cdot(\varepsilon y) .
\end{aligned}
$$

Thus $a \cdot b \in Q_{\lambda}(\cdot)$. Also

$$
\begin{aligned}
a\left(a^{\rho} x \cdot y\right) & =\left(a \cdot a^{\rho} x\right) \cdot(\varepsilon y)=x \cdot \varepsilon y \quad \text { by }(3) \\
& =\varepsilon(\varepsilon x \cdot y)=\left(a a^{\rho}\right) \cdot(\varepsilon x \cdot y)=a\left(a^{\rho}(\varepsilon(\varepsilon x \cdot y))\right)=a\left(a^{\rho}(x \cdot \varepsilon y)\right) ;
\end{aligned}
$$

by the left cancellative law $a^{\rho} x \cdot y=a^{\rho}(x \cdot \varepsilon y) \Rightarrow a^{\rho} \in Q_{\lambda}(\cdot)$. Thus $Q_{\lambda}(\cdot)$ is a sub-left loop of $Q(\cdot)$. Let $a \in Q_{\lambda}(\cdot) \cap N_{\lambda}(\cdot)$; then $a \cdot x y=a x \cdot y$ and $a x \cdot \varepsilon y$ $=a \cdot x y$ for all $x, y \in Q$. Thus we have $a x \cdot y=a x \cdot \varepsilon y$ by the left cancellative law, $y=\varepsilon y$ for all $y \in Q$. It is a contradiction and hence $N_{\lambda}(\cdot) \cap Q_{\lambda}(\cdot)$ $=\varnothing$.

Similarly we can prove the results for $N_{\mu}(\cdot)$ and $Q_{\mu}(\cdot)$. From (22) and (c), we get $N_{\rho}(\cdot)=\left\{\right.$ all $a \in Q_{\rho}(\cdot) \mid \varepsilon \cdot a=a$ for all $\left.a \in Q_{\rho}(\cdot)\right\}$. It implies that $N_{\rho}(\cdot)$ $\subset Q_{\rho}(\cdot)$.

THEOREM 7. If $Q(\cdot)$ is a left loop with L.B.P. and satisfies the identity $(x \cdot y)^{\rho}=y^{\rho} \cdot x^{\rho}$ for all $x, y \in Q$, then $Q(\cdot)$ is a Moufang loop.

Proof. $(x \cdot y)^{\rho}=y^{\rho} \cdot x^{\rho} \Rightarrow(\varepsilon \cdot y)^{\rho}=y^{\rho}$. But $(\varepsilon \cdot y)^{\rho}=\varepsilon \cdot y^{\rho}$. Consequently $\varepsilon \cdot y^{\rho}=y^{\rho}$ for all $y \in Q$ which implies $\varepsilon \cdot z=z$ for all $z \in Q$, and the right identity $\varepsilon$ is left as well. From Theorem 1(ii),

$$
\begin{aligned}
x^{\rho} \cdot y^{\rho} x^{\rho} & =(x \cdot y x)^{\rho}=(y x)^{\rho} \cdot x^{\rho}=x^{\rho} y^{\rho} \cdot x^{\rho} \\
& \Rightarrow x \cdot y x=x y \cdot x \quad \text { for all } x, y \in Q .
\end{aligned}
$$

For all $x, y, z \in Q$,

$$
\begin{aligned}
x(y \cdot x z) & =(x \cdot y x) z & \text { by }(2) \\
& =(x y \cdot x) z & \text { by }(23) .
\end{aligned}
$$

Thus $Q(\cdot)$ satisfies the Moufang identity. Hence, by Bruck's Lemma [4, Lemma 3.1, p. 115], $Q(\cdot)$ is a Moufang loop. 
Corollary 3. If $Q(\cdot)$ is a left loop with L.B.P. and diassociative, then it is a Moufang loop.

The proof is obvious from Theorem 7.

THEOREM 8. If $Q(\cdot)$ is a left loop with L.B.P. and is right alternative, then it is a Moufang loop.

Proof. Since $Q(\cdot)$ is right alternative, $x y \cdot y=x \cdot y y$ for all $x, y \in Q$. Therefore, $y(x y \cdot y)=y(x \cdot y y)=(y \cdot x y) y$ by (2). Put $x y=z$. Thus $y \cdot z y$ $=y z \cdot y$ for all $y, z \in Q$. The rest of the proof follows from Theorem 7 .

THEOREM 9. If $Q(\cdot)$ is a left loop with L.B.P. and R.B.P., then it is a Moufang loop.

Proof. Since $Q(\cdot)$ satisfies (1) and by putting $z=\varepsilon$ in (1), we have

$$
x y \cdot y=x \cdot y y \text { for all } x, y \in Q .
$$

Thus $Q(\cdot)$ is right alternative. Hence $Q(\cdot)$ is a Moufang loop by Theorem 8 .

ACKNOWLEDGEMENT. The author is thankful to the referee for constructive criticism and helpful comments.

\section{REFERENCES}

1. B. Bol, Gewebe und Gruppen, Math. Ann. 114 (1937), 414-431.

2. D. A. Robinson, Bol loops, Trans. Amer. Math. Soc. 123 (1966), 341-354. MR 33 \#2755.

3. R. H. Bruck, Contributions to the theory of loops, Trans. Amer. Math. Soc. 60 (1946), 245-354. MR 8, 134.

4. — , A survey of binary systems, Springer-Verlag, New York and Berlin, 1958. MR 20 \#76.

Department of Mathematics, University of Ife, Ile-Ife, Nigeria

Current address: Department of Mathematics, University of the West Indies, Kingston 7, Jamaica, West Indies 